\title{
Cooperative Localization For Autonomous Vehicles Sharing GNSS Measurements
}


- 


\section{List of Figures}

1.1 Illustration of the effects of biased measurements. Confidence domains are zones in which the vehicle is highly located. . . 29

1.2 Experimental vehicles and shared data . . . . . . . 29

1.3 Bracketing of the solution set $\mathbb{X}$ between two subpavings $\underline{\mathbb{X}}$ and $\overline{\mathbb{X}}=\underline{\mathbb{X}} \cup \Delta \mathbb{X} .[\overline{\mathbb{X}}]$ is the hull box of $\overline{\mathbb{X}}$. . . . . . . . .

1.4 Trajectories of both vehicles when using C-SIVIAP. Reference and position boxes are displayed. The mean speed of vehicles was $30 \mathrm{Kmph}$. Every vehicle did several loops of its trajectory.

1.5 Bounds of position errors of $R_{1}$ centered on the reference. . . 31

1.6 Bounds of position errors of $R_{2}$ centered on the reference. . .

1.7 Estimates and bounds of pseudoranges biases (units are samples and meters). . . . . . . . . . . . . . . . 32

1.8 HPE and CDS illustration for C-EKF-CI. . . . . . . . . 32

1.9 HPE and $k \sigma_{H P E}$ illustration for C-SIVIAP. . . . . . . . 32

1.10 Plots of the cumulative distribution function (CDF) of the HPE produced by the two methods in the two vehicles (C EKF : cooperative EKF based on CI fusion, C SIVIA : cooperative set-membership method). . . . . . . . . . . . .

1.11 simplified Stanford Diagram : Filters consistency when using cooperative set-membership and Bayesian methods for $R_{1}$ and $R_{2}$. (a) C-SIVIAP for $R_{1}$, (b) C-EKF based on CI fusion for $R_{1}$, (c) C-SIVIAP for $R_{2}$, (d) C-EKF based on CI fusion for $R_{2}$. . 
- 


\section{List of Tables}

1.1 Noises parameters used for the estimation methods. . . . . . 17

$1.2 \mathrm{HPE}$, relative distance and CDS statistics. . . . . . . . . 18 
- 


\section{1 \\ Cooperative Localization For Autonomous Vehicles sharing GNSS measurements}

\section{CONTENTS}

1.1 Introduction: State of the art of Cooperative Localization for

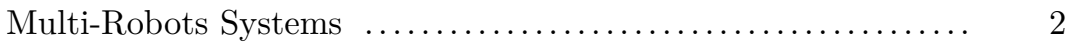

1.1.1 Centralized Cooperative Estimation $\ldots \ldots \ldots \ldots \ldots \ldots . . \ldots 2$

1.1.2 Decentralized Cooperative Estimation $\ldots . \ldots \ldots \ldots \ldots . . . .4$

1.1.3 Common Methods for Estimation (i.e. Localization) ... 6

1.1.4 Technologies for Positioning $\ldots \ldots \ldots \ldots \ldots \ldots \ldots \ldots . \ldots . \ldots . \ldots 6$

1.2 System Modeling and Observability Analysis For Cooperative

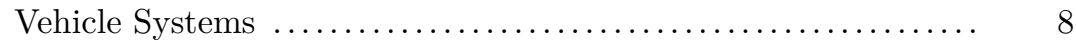

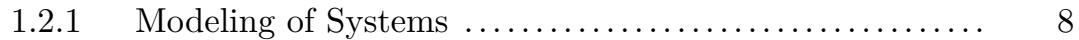

1.2.1.1 Sensor Model or Distances (i.e.

Pseudo-distances) Measurements ......... 8

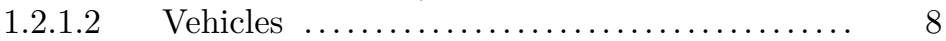

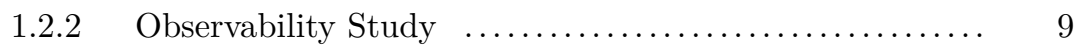

1.2.2.1 Definitions and theorems $\ldots \ldots \ldots \ldots \ldots \ldots . . \ldots$

1.2.2.2 Dynamic case study .................. 10

1.3 Methodology for Cooperative State Estimation $\quad \ldots . \ldots . \ldots \ldots \ldots \quad 12$

1.3.1 Computing Reliable Confidence Domains with a Set Membership Method ...................... 13

1.3.1.1 Set Inversion with Constraints Propagation . 13

1.3.2 Cooperative Localization Problem Statement ......... 14

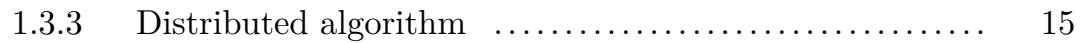

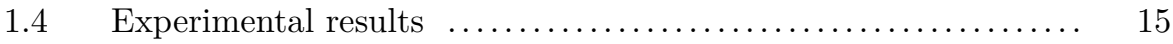

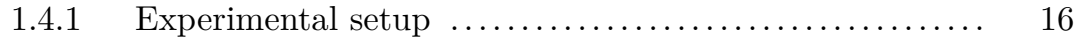

1.4.2 Set-membership method (SIVIAP) performance

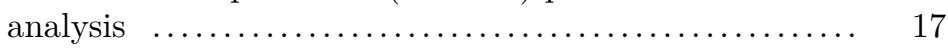

1.5 Comparison with a distributed Extended Kalman filtering implementing a covariance intersection $\ldots \ldots \ldots \ldots \ldots \ldots \ldots \ldots . \ldots . \ldots . \ldots . \ldots 19$

1.5.1 Backgrounds for Comparison of both methods ....... 19

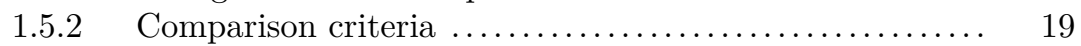

1.5.2.1 Horizontal Protection Errors ............. 20

1.5.2.2 Confidence Domain Size ................. 20

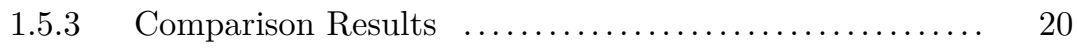


2 Cooperative Localization For Autonomous Vehicles sharing GNSS measurements

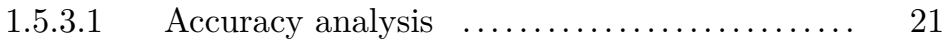

1.5.3.2 Consistency analysis $\ldots \ldots \ldots \ldots \ldots \ldots \ldots . . \ldots 21$

1.6

Conclusion

22

\subsection{Introduction: State of the art of Cooperative Local- ization for Multi-Robots Systems}

In this Chapter, we propose a new formulation of multi-vehicles cooperation based on sharing GNSS corrections, as a way to extend the principle of Differential GPS (DGPS) to dynamic reference base stations. We present a new cooperative algorithm for vehicles positioning based on set inversion method with Constraint Satisfaction Problem (CSP) techniques on intervals. When using V2X communications, vehicles cooperate and exchange information such that each vehicle can compute the positions of the partners with a reliable domain. The ego-motion of every vehicle can also be used to improve the cooperation. The proposed method relies on the exchange of positions estimates, pseudorange estimated errors and Dead-Reckoning (DR) data (see Fig. 1.2). A first contribution of this work is to show that it is possible to improve the ego positioning and mutual localization between vehicles by sharing GNSS biased pseudoranges using a model of the correlation of the pseudorange errors and through the knowledge of the local motions of the vehicles done by DR or tracking. In the following, an introduction provides an overview of the most recent developments or work in cooperative localization over the last decade. In particular, we will introduce four variants in the architecture of cooperative localization. The section 1.2 will introduce a system modeling and an observability study of the cooperative system. Then, in section 1.3, the proposed distributed estimation method is presented. It allows the data fusion of the estimated biases in a distributed way with no central fusion node and with no base station. An experimental evaluation with two vehicles is performed in section 1.4 to evaluate the performance in real conditions using a ground truth system. The performance of the cooperation is clearly highlighted. A classical sequential Bayesian method has also been implemented on the same data set and is compared in terms of accuracy and confidence.

\subsubsection{Centralized Cooperative Estimation}

There are two architectures of cooperative systems: centralized and decentralized. Many variants of cooperative positioning systems are essentially based on centralized systems. Centralized system performs state estimation using a central,fixed unit. This centre communicates with all robots of the group. The principle of centralized estimation includes the state estimation of each robot in a unique state vector of the group. The update of the state of each 
Introduction: State of the art of Cooperative Localization for Multi-Robots Systems 3

robot is performed through treatment of sensor data collected from each robot. This data can be egocentric (i.e. proprioceptive) or observational (i.e. exteroceptive). Egocentric data includes robots motions or their absolute pose. Observational data is can be inter-robot or environmental or external data, such as the relative pose between robots or the range between robots and obstacles.

Hereafter, a state of the art in cooperative localization through centralised architecture is given.

The most significant developments or progress in cooperative positioning has been achieved by Roumeliotis and Bekey [1], who presented a cooperative system for positioning of a fleet of mobile robots using a centralized approach. In this approach, they used an Extended Kalman Filter to update the state of the fleet. Several cooperative approaches have extended Roumeliotis and Bekeys research to study, for example, the impacts of using relative data such as orientation measurements and relative distances in the fleets state update process [2] or furthermore, the propagation of uncertainties [3]. Data fusion methods are not limited to the Kalman filter; other tools are proposed, such as the Posterior Maximum Estimator [4] and the Maximum Likelihood Estimation [5]. Some applications use other types of sensors for example other research proposes to update the robots states using acoustic or visual sensors. A camera that measure relative orientation was used in a cooperative positioning method in [6]. Acoustic waves were used to measure the relative pose between robots [7].

Centralized approaches have advantages, such as the simplicity of the centralized merger of the data collected from each robot, allowing for an optimal update of the state of the group. The interdependencies are handled in a natural way as the data are only merged once time in the CPU, so there is no risk of the reuse of an identical information in the merge process. However, in practice, centralized approaches have disadvantages related to the communication constraints with the central unit. The robots have to maintain permanent contact with the infrastructure. They must be close since the range of the communication systems is often short since it depends on deployment provided by the phone provider, which limits the robots area of evolution. The more remote the network access point, the greater the loss in energy level of data transmission. The rate of transmission decreases also with more data users in the same area. In order to overcome these disadvantages, the centralized architecture has evolved into a multi-centralized architecture. As a way to expand the robots area of evolution, mobile agents gathering information are used as intermediaries between the mobile robots and the infrastructure. In the multi-centralized localization context, Worrall and Nebot have used multi-hop communications to transmit real-time vehicle state information to other vehicles [8]. However, an isolated agent problem occurs when the environment is large and sparsely populated. One possible solution to this problem is to use additional mobile agents that navigate near the isolated agent to collect updates of state information and bring them to 
the main network [9]. However, in these proposed solutions, the centralized system always depends on the infrastructure and therefore a failure of the infrastructure impacts the whole system. Given the technological evolution and the availability of embedded computers in several kinds of robots, researchers have started to examine how to get rid of the central system and to distribute the estimated pose of the group within its members. This type of architecture is called distributed or decentralized architecture.

\subsubsection{Decentralized Cooperative Estimation}

In a distributed fusion system, each vehicle processes its measurements and communicates the results with the other vehicles. The distributed architecture does not have a central server sharing information with the members of the group. The basic concept of distributed cooperative localization is the same as the centralized approaches.

The vehicles in the group are considered as a system of systems that uses data from each member. However, in this case each agent has a version of the state of the group which it updates with its own information and with the shared data from its partners. This kind of architecture was introduced by Roumeliotis et al [10]. The authors propose a cooperative localization system based on an Extended Kalman Filter. This approach is based on the modification of the Kalman Filter equations to distribute them to the different members of the group. This method enables the production of the same result as a centralized estimator. Other works have been inspired by this approach and propose solutions based on different sensors and mathematical tools for fully distributed information fusion. The distributed approaches can be divided into two broad categories according to the type of shared data between vehicles :

- Approaches based on the exchange of sensor measurements.

- Approaches based on the exchange of fused states or parts of them.

In the following, each type of approach is described by providing some examples from the literature. The concept of sensor-based exchange approaches is that each vehicle has to send its measurements to all its partners in order to have an updated version of the status in each vehicle.

For example, Hery et al. [11] proposed a cooperative localization method based on state exchange to improve the along-track position of vehicles. The vehicles are using relative measurements from perception sensors such as Lidars. They introduced a one-dimensional formulation of the localization problem under the hypothesis that lateral distances and headings of vehicles are well known. Madhavan et al. presented a distributed system of cooperative localization of heterogeneous mobile robots in [12]. The proposed approach has been applied on a fleet of two mobile robots. The cooperation is carried 
out by shared information about the relative pose that contains the distances between both robots, the relative orientation, and the absolute position, which contains GPS and proprioceptive measurements. Thanks to the exchange of data, each robot has a state version of the group. In this case, the need for permanent availability of communication between the robots is essential. Moreover, the amount of data to be shared increases according to the number of robots used in the group and the sensor rates. The estimates produced by each robot are suboptimal compared to a centralized estimator. Fox et al. [13] have adopted a similar approach to [12] in the sense that each robot has to maintain an estimate of its own pose. They used a Particle Filter method instead of an Extended Kalman Filter and a density tree approach for the fusion of the relative shared data. This approach temporarily combines the probability density functions (i.e, particles) from both robots to perform a sampling of importance.

In fused state exchange approaches, each robot only uses its own sensors to update the state of the group and only shares its final result. This type of approach limits the amount of exchanged data. However, these approaches suffer from the problem of data incest. This problem is due in particular to the re-use of the same information in the fusion process. The estimates obtained after the fusion in this case are biased and overconfident. Indeed, the problem of data consanguinity generates a problem of over-convergence which comes from the ignorance of the correlations between the estimates of robots. Several solutions to the data incest problem have been proposed. For example, Howard et al. have used an approach based on the exchange of measured pose distributions, where each robot estimates the positions of the others robots in the group regarding its own position using the measurements of a camera and a laser ranging [14]. The fusion of shared information is performed using a Particle Filter. After the data exchange, each robot updates the distribution of its pose with the one received from the detected robot. In order to avoid the problem of circular updates, the author keeps up to date a dependency tree in order to only update the descendants with data that has not been merged before. Other solutions are proposed by Roumeliotis et al. and Karam et al. $[15,3]$. In [3], robots only share a part of their state and, during the fusion process, only a part of the collected information is considered. In this case, the obtained fused state contains suboptimal estimates since we do not use all the shared information. In [15], the overall state of the system combines all local states collected by all members of the group and the global state is only used by local robots but never communicated again to other members.

However, this work does not address the consistency / integrity of the estimates. The covariance intersection method represents a solution of the data incest problem since it manages the dependencies between estimates and at the same time, offers reliable confidence domains. The above-mentioned methods are all based on Bayesian approaches (Extended Kalman Filter) or Particle Filter. Other fusion approaches can avoid the problem of over-convergence while guaranteeing results with reliable confidence domains, such as set-membership 
6 Cooperative Localization For Autonomous Vehicles sharing GNSS measurements

approaches $[16,17,18,19]$. This will be the following approach in this study, in particular the set-membership method based on interval analysis.

\subsubsection{Common Methods for Estimation (i.e. Localization)}

The set-membership approach is based on the assumption that errors of models and measurements are bounded. It has been successfully applied for the estimation of model parameters [20] and the estimation of robot positions when reliable confidence domains are required [21]. Meizel et al. [22] developed a Set Inversion method by using Interval Analysis techniques (SIVIA) based on bounded error observers for the localization of one robot. However, SIVIA is not suitable for real-time applications when the initial area of research is too large, which leads to increase the number of bisections in the computation process. One solution is to use SIVIA while simultaneously solving a constraint satisfaction problem (CSP) to limit the calculation time of bisections [23]. Regarding real-time cooperative localization, several recent studies based on set-inversion with the CSP techniques were studied. Drevelle et al. [24] operated a group of autonomous underwater vehicles (UAV) to explore a large area. In their application, they used ranging sensors to measure the inter-distance between robots.

In addition, Bethencourt [24] used distributed set-membership algorithms to accomplish a cooperative mission of a group of UAVs using inter-temporal measurements. Kyoung-Hwan and Jihong also studied a cooperative localization method for several ground robots based on constraint propagation techniques [25]. In this work, the fusion of proprioceptive and exteroceptive sensors data was performed. In summary, considerable work has been done in simulation of cooperative systems, but very few results using real data have been reported. Finally, little work has been done to evaluate the performance of the integrity of estimates from low-cost sensors.

\subsubsection{Technologies for Positioning}

Localization is used in many fields, such as by sailors who need to know their absolute position regularly. Today, many consumer applications have appeared which utilize localization. The list of applications is very wide and new uses have appeared regularly in recent years. In the field of robotics, localization can guide robots in difficult places that are inaccessible or contaminated to perform different tasks autonomously. In Intelligent Transportation Systems (ITS), localization is an essential task for the navigation of intelligent vehicles. Whether localization is employed to inform the user of his position in order to guide him, or for autonomous control, the vehicle must be able to localize itself in its environment.

There are different technologies for positioning mobile robots in a free space. They are based on communicating systems that use transmitters (e.g. ground 
infrastructure such as beacons) and receivers (e.g. GPS, UWB: Ultra Wide Band, WiFi, Bluetooth, RFID, etc.), or on embedded sensors for navigation (e.g. MEMS, camera, etc) without relying on infrastructure or the combination of communicating systems with autonomous sensors. Among the most widespread technologies based on ground infrastructure, there are systems using radio positioning techniques based on phone networks or wireless networks (e.g. UWB, WiFi, etc..), where the mobile object is localized using the signals it transmits to the network (e.g. $2 \mathrm{G}, 3 \mathrm{G}, \mathrm{WiFi}$, RFID etc..) or receives from base stations or access points in that network. The main disadvantage of these methods is that their coverage area is limited. Geopositioning by satellite navigation systems (e.g. GNSS: Global Navigation Satellite System) offers the benefit of global coverage with a constellation of satellites in orbit (although some systems, based on geostationary satellites, have only regional coverage). Since the first satellite system, Global TRANSIT commissioned in 1967, other systems have been developed. The NAVSTAR GPS, GALILEO, GLONASS and BEIDOU systems are thus currently operational or being deployed. These systems provide absolute positioning with an accuracy of ten meters, for a reasonable cost. However, satellite techniques are not effective in urban environments and closed environments causing errors of several dozen meters. An alternative method is the hybridization of GNSS positioning with other sources of information such as inertial measurements (e.g. speed and orientation measurements), relative location based on the detection of beacons (e.g.UWB, radar) or geometric elements of the route (e.g a map). Dead Reckoning (DR) sensors and inertial sensors are frequently used as alternative technologies for determining position, speed and attitude. They are very common and measurements can easily be collected, for example from a CAN bus embedded on a car.

We are interested in a hybrid method that combines ground and satellite radionavigation systems with DR sensors. The technologies considered in this chapter for the positioning of vehicles are the fusion of DR measurements with radio navigation measurements such as GNSS pseudorange measurements. In the following, a mutual cooperation through information exchange is used to enhance positioning accuracy of a group of autonomous vehicles. We aim also to reduce uncertainty arising from the use of low cost sensors. For example, mono-frequency Global Navigation Satellite Systems (GNSS) receivers in complex environments usually leads to offsets between real and observed positions (Fig. 1.1). These systematic offsets (i.e, biases) are often due to inaccurate satellite positions, atmospheric and tropospheric errors. The impact of these biases on vehicles localization should not be neglected. Cooperation and exchange of biases estimates between vehicles can reduce significantly these systematic errors [26]. However, distributed cooperative localization based on sharing estimates is subject to data incest problems. When position information is used in a safety-critical context (e.g. autonomous vehicles navigation in proximity), one should guaranty the consistency of the localization estimates. In this context, we mainly aim to improve the absolute and rela- 
8 Cooperative Localization For Autonomous Vehicles sharing GNSS measurements

tive performances of vehicles localization through cooperation. Moreover, we focus on characterizing reliable confidence domains (see Fig. 1.1) that contain vehicles positions with high reliability.

\subsection{System Modeling and Observability Analysis For Cooperative Vehicle Systems}

Here we present the mathematical models used to determine the positions of autonomous systems. We will present basic models which are the most common in robotic applications. Then, we will present a dynamic observability study based on Lie Derivatives. Observability studies are essential step for the localization of vehicles. One should check if the vehicles positions and measurements biases can be determined before the development of any observer.

\subsubsection{Modeling of Systems}

\subsubsection{Sensor Model or Distances (i.e. Pseudo-distances) Mea- surements}

The GNSS raw measurements considered here are $L 1$ pseudoranges. The pseudorange $\rho_{i}^{j}[27]$ is the measure of each vehicle $R_{i}\left(i=\left\{1, \ldots, n_{r}\right\}\right)$ located at coordinates $\boldsymbol{p}_{i}=\left[x_{i}, y_{i}, z_{i}\right]$ to each satellite $j\left(j=1, \ldots, n_{s}\right)$ at position $\boldsymbol{p}^{j}=\left[x^{j}, y^{j}, z^{j}\right]$. We model the common GNSS errors $b^{j}$ as additive errors on the pseudoranges. The model of $\rho_{i}^{j}$ is expressed in Eq. (1.1). Please refer to [28] for further details.

$$
\rho_{i}^{j}=\sqrt{\left(x_{i}-x_{j}\right)^{2}+\left(y_{i}-y_{j}\right)^{2}+\left(z_{i}-z_{j}\right)^{2}}+b^{j}+d_{i}+\beta^{j}
$$

where $d_{i}$ represents the receiver clock offset and $\beta^{j}$ the measurement noise.

Let ${ }^{i} \boldsymbol{y} \in \mathbb{R}^{n_{s}}$ be the vector of $n_{s}$ pseudoranges measurements and ${ }^{i} \boldsymbol{x} \in \mathbb{R}^{n}$ be the state vector of vehicle $i$. The observation model at discrete time $k$ is defined as follows

$$
{ }^{i} \boldsymbol{y}_{k}=\boldsymbol{g}\left({ }^{i} \boldsymbol{x}_{k}\right)
$$

Suppose that each vehicle is equipped with a GNSS receiver and DR sensors that provide speed and heading in an input $\operatorname{vector}{ }^{i} \boldsymbol{u}(t)=\left[\begin{array}{ll}v_{i} & \psi_{i}\end{array}\right]^{T}$. 
System Modeling and Observability Analysis For Cooperative Vehicle Systems 9

\subsubsection{Vehicles}

A 2D unicycle evolution model for the pose components, a linear model for the receiver clock offset and an auto-regressive (AR) model for the pseudoranges errors are concatenated and described by a continuous function $\boldsymbol{f}$ in a local East North Up frame (time $t$ is omitted for clarity):

$$
\boldsymbol{f}\left({ }^{i} \boldsymbol{x},{ }^{i} \boldsymbol{u}\right)=\left\{\begin{array}{l}
\dot{x}_{i}=v_{i} \cos \left(\psi_{i}\right) ; \quad \dot{y}_{i}=v_{i} \sin \left(\psi_{i}\right) ; \quad \dot{z}_{i}=0 \\
\dot{d}_{i}=d r_{i} ; \quad \dot{d} \dot{r}_{i}=0 ; \quad \dot{b}^{j}=a b^{j}
\end{array}\right.
$$

$d_{i}$ and $d r_{i}$ are respectively the receiver clock offset and its drift to be estimated. The AR parameter is $a=e^{-T e / \tau}, T_{e}$ being the sampling time and $\tau$ the time constant of the model bias $b^{j}(a=0.9995, \tau=6.2 \mathrm{~min})$. The indexes of the vehicles and common satellites are respectively denoted by $i \in\left\{1 \ldots n_{r}\right\}$ and $j \in\left\{1 \ldots n_{s}\right\}$.

\subsubsection{Observability Study}

\subsubsection{Definitions and theorems}

Errors on pseudorange measurements are spatially correlated and similar for nearby users [27] but not directly observed with no base station. Therefore, it is essential to study the observability to evaluate if the problem is solvable. Rife and Xiao [29] have shown that it is not possible to estimate biases simply by sharing GNSS pseudorange measurements between vehicles communicating via a Vehicle-to-Vehicle (V2V) network in a snapshot way (Epoch by Epoch). They highlighted the limitation of distributing only GNSS data and proposed to add georeferenced information by using camera-based lane boundary sensor. A natural question that arises is whether GNSS biases are observable when adding vehicles motion information and errors evolution model.

In this section, we investigate the observability of the cooperative localization problem of vehicles sharing biases estimates when they are moving. The cooperative system described in section 1.2.1 given DR and pseudorange measurements is nonlinear. Therefore, we use the observability rank criterion based on Lie-Derivatives [30] to determine the conditions under which the system is locally weakly observable. Note that Martinelli and Siegwart [31] have employed this criterion to investigate the observability of $2 \mathrm{D}$ cooperative localization of mobile robots. Recently, Zhou et al. [32] have used the Lie derivatives to determine the conditions for the observability of $2 \mathrm{D}$ relative pose of pairs of mobile robots using range measurements. In the sequel, a test of Lie derivatives is considered for vehicles sharing GNSS errors. This study is inspired by the work of Zhou et al. [32].

Definition 1. (Observability Rank Condition): The observability rank condition is satisfied when the observability matrix is full rank. 
10Cooperative Localization For Autonomous Vehicles sharing GNSS measurements

Theorem 1. (Observability Sufficient Condition): If the system satisfies the observability rank condition at a given state $\boldsymbol{x}^{0}$ (at some time), then the system is locally weakly observable at $\boldsymbol{x}^{0}$ [30].

\subsubsection{Dynamic case study}

Let consider $n_{r}$ vehicles and $n_{s}$ common visible satellites. Let $\hat{\boldsymbol{x}}$ be the estimated state of the cooperative system (S) as follows

$$
\hat{\boldsymbol{x}}=\left[\hat{\boldsymbol{p}}_{1}, \hat{d}_{1}, \ldots, \hat{\boldsymbol{p}}_{n_{r}}, \hat{d}_{n_{r}}, \hat{b}^{1}, \ldots, \hat{b}^{n_{s}}\right]^{T}
$$

with $\operatorname{dim}(\hat{\boldsymbol{x}})=4 n_{r}+n_{s} . \quad \hat{\boldsymbol{p}}_{1 \cdots n_{r}}$ are the $3 \mathrm{D}$ vehicles positions, $\hat{d}_{1 \cdots n_{r}}$ represent the receivers clock offsets. $\left(\hat{b}^{1 \cdots n_{s}}\right)$ denote the biases on $n_{s}$ common pseudorange measurements between vehicles.

The considered evolution model in this study consists in the first four DR equations of the system (1.3) and the last equation for the evolution of biases. Let consider $u=\left[v_{1} \ldots v_{n_{r}}\right]^{T}$ the input of the system (S) in (1.4). The nonlinear DR model of (S) can be written as follows

$$
\dot{\boldsymbol{x}}=\overbrace{\left[\begin{array}{c}
0 \\
0 \\
0 \\
d r_{1} \\
\vdots \\
0 \\
0 \\
0 \\
d r_{n_{r}} \\
a b^{1} \\
\vdots \\
a b^{n_{s}}
\end{array}\right]}^{f_{0}}+\overbrace{\left[\begin{array}{c}
\cos \left(\psi_{1}\right) \\
\sin \left(\psi_{1}\right) \\
0 \\
0 \\
\vdots \\
0 \\
0 \\
0 \\
0 \\
0 \\
\vdots \\
0
\end{array}\right]}^{f_{1}} v_{1}+\cdots+\left[\begin{array}{c}
0 \\
0 \\
0 \\
0 \\
\cos \left(\psi_{n_{r}}\right) \\
\sin \left(\psi_{n_{r}}\right) \\
0 \\
0 \\
0 \\
\vdots \\
0
\end{array}\right] v_{n_{r}}^{f_{n_{r}}}
$$

The nonlinear observation equations are given by

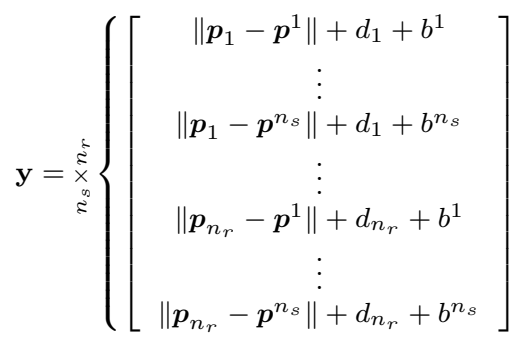

We compute hereafter the necessary Lie derivatives of $\mathbf{y}$ and their gradients: 
System Modeling and Observability Analysis For Cooperative Vehicle Systems 11

Zeroth-order Lie derivatives $\left(\mathcal{L}^{0} \mathrm{y}\right)$ :

$$
\mathcal{L}^{0} \mathbf{y}=\mathbf{y}
$$

with gradient:

$$
\begin{aligned}
& \nabla \mathcal{L}^{0} \mathbf{y}=j \operatorname{acobian}(\mathbf{y})
\end{aligned}
$$

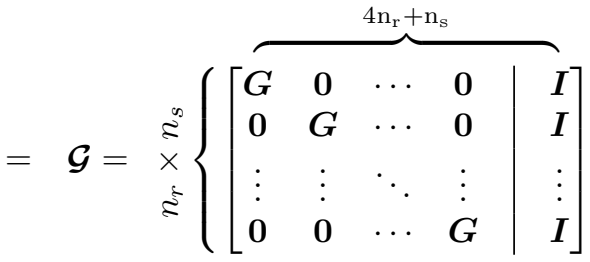

where $\boldsymbol{I}$ is the identity matrix with $\operatorname{dim}(\boldsymbol{I})=n_{s} \times n_{s}$ and $\mathcal{G}$ is the geometry matrix described in [29] where $\boldsymbol{G}$ is defined as follows:

$$
\boldsymbol{G}=\left[\begin{array}{cc}
\left(\boldsymbol{u}^{1}\right)^{T} & 1 \\
\left(\boldsymbol{u}^{2}\right)^{T} & 1 \\
\vdots & \vdots \\
\left(\boldsymbol{u}^{n_{s}}\right)^{T} & 1
\end{array}\right]
$$

with $\operatorname{dim}\left(\boldsymbol{G}_{i}\right)=n_{s} \times 4$, the unit vector $\boldsymbol{u}^{j}$ in $\boldsymbol{G}$ is the estimated line of sight from the satellite $j$ to each user receiver $i$. This pointing vector is the same for all users when they are assumed to be in close proximity (i.e. distance between vehicles $\leq 10 \mathrm{~km})$ :

$$
\boldsymbol{u}^{j}=\left(\boldsymbol{p}_{i}-\boldsymbol{p}^{j}\right) /\left\|\boldsymbol{p}_{i}-\boldsymbol{p}^{j}\right\|
$$

First-order Lie derivatives $\left(\mathcal{L}_{f_{0}}^{1} \mathrm{y}\right)$ :

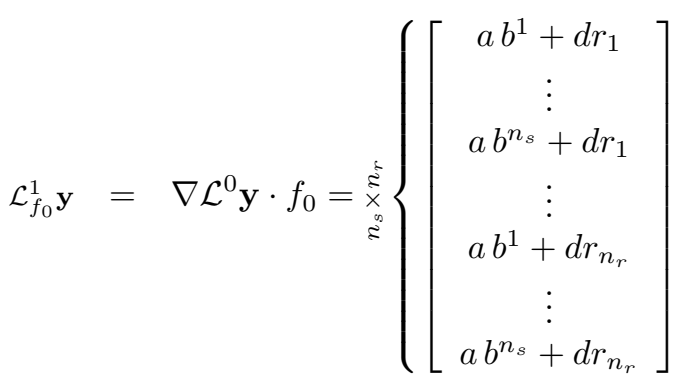

with gradient: 
12 Cooperative Localization For Autonomous Vehicles sharing GNSS measurements

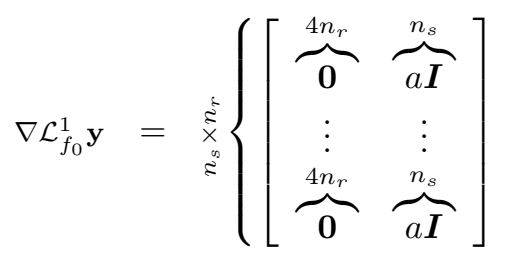

The observability matrix is now:

$$
\mathcal{O}=\left[\begin{array}{c}
\nabla \mathcal{L}^{0} \mathbf{y} \\
\nabla \mathcal{L}_{f_{0}}^{1} \mathbf{y}
\end{array}\right]
$$

The role of the matrix in Eq. (1.10) in the observability analysis of a nonlinear system is given in [30], and recalled in definition 1 and theorem 1.

Below, we compute the rank of the observability matrix (1.10) and determine the necessary conditions under which the system (S) can be locally weakly observable. Here, we have:

$$
\operatorname{rank}(\mathcal{O})=\operatorname{rank}\left(\nabla \mathcal{L}^{0} \mathbf{y}\right)+\operatorname{rank}\left(\nabla \mathcal{L}_{f_{0}}^{1} \mathbf{y}\right) .
$$

The rank of $\nabla \mathcal{L}^{0} \mathbf{y}$ has been studied in [29]. They proved that $\operatorname{rank}\left(\nabla \mathcal{L}^{0} \mathbf{y}\right)=$ $4\left(n_{r}-1\right)+n_{s}$. It is straightforward to determine the rank of $\nabla \mathcal{L}_{f_{0}}^{1} \mathbf{y}$. Since the number of linearly independent equations in $\nabla \mathcal{L}_{f_{0}}^{1} \mathbf{y}$ appears to be $n_{s}$ (i.e $\left.\operatorname{rank}\left(\nabla \mathcal{L}_{f_{0}}^{1} \mathbf{y}\right)=n_{s}\right)$ if we have $a \neq 0$ (i.e. auto-regressive model of the biases). So, according to (1.11) we get:

$$
\operatorname{rank}(\mathcal{O})=4\left(n_{r}-1\right)+2 n_{s}
$$

In order to get a full rank of $\mathcal{O}$, one must discuss the least required number of $n_{s}$ common satellites between users. It is obvious that $\operatorname{rank}(\mathcal{O})$ can not exceed the $n_{I}$ unknowns states of $(\mathrm{S})$ which is equal to $4 n_{r}+n_{s}$ (i.e. $\operatorname{rank}(\mathcal{O}) \leq$ $\left.4 n_{r}+n_{s}\right)$, so to get a full rank of $\mathcal{O}$ one must determine $n_{s}$ such that

$$
\operatorname{rank}(\mathcal{O}) \geq 4 n_{r}+n_{s} .
$$

By replacing (1.12) in (1.13) we get: $n_{s} \geq 4$. The observability rank condition (definition 1) is obtained when this condition is satisfied. According to theorem 1, it can be concluded that the system is locally weakly observable regardless the number of users $\left(n_{r}\right)$ if the biases have an auto-regressive behavior and at least 4 common satellites between the vehicles. Please note also that, as we have only used the Lie derivative with respect to $f_{0}$, the system is observable even if the the vehicles are motionless. 


\subsection{Methodology for Cooperative State Estimation}

This section will introduce how to extend non-cooperative methods of localization to cooperative ones. Essentially, there are two methods: the probabilistic (or Bayesian) method and the bounded error (or set-membership) method.

\subsubsection{Computing Reliable Confidence Domains with a Set Membership Method}

\subsubsection{Set Inversion with Constraints Propagation}

To perform a state estimation in a bounded error framework with intervals, one needs solving a set inversion problem. The objective is to determine the unknown state $\mathbb{X} \subset \mathbb{R}^{n}$ such as $\mathbf{f}(\mathbb{X}) \subset \mathbb{Y}$, where $\mathbb{Y}$ is the known set of measurements. The objective is to compute the reciprocal image $\mathbb{X}=\mathbf{f}^{-1}(\mathbb{Y})$. A guaranteed approximation of the solution set $\mathbb{X}$ can be done using 2 subpavings which bracket the solution set as follows: $\mathbb{X} \subset \mathbb{X} \subset \overline{\mathbb{X}}$ (Fig. 1.3).

A box $[\mathbf{x}]$ of $\mathbb{R}^{n}$ is feasible if it is inside $\mathbb{X}$ and unfeasible if it is outside $\mathbb{X}$, otherwise $[\mathbf{x}]$ is indeterminate. By using an inclusion function $[\mathbf{f}]$ of function $f$, one can identify the feasibility of the boxes using the following tests:

- If $[\mathbf{f}]([\mathbf{x}]) \subset \mathbb{Y}$ then $[\mathbf{x}]$ is feasible

- If $[\mathbf{f}]([\mathbf{x}]) \cap \mathbb{Y}=\emptyset$ then $[\mathbf{x}]$ is unfeasible

- Else $[\mathbf{x}]$ is indeterminate.

SIVIA solves the set inversion problem by testing recursively the feasibility of candidate boxes, starting from an arbitrarily large initial box $\left[\mathbf{x}_{\mathbf{0}}\right][20]$. If a box is feasible, it is stored in the inner solution set $\underline{\mathbb{X}}$. If a box is unfeasible, it is discarded since the resulting box $([\mathbf{f}]([\mathbf{x}]))$ is outside the measurement domain $\mathbb{Y}$. Finally, an indeterminate box is bisected into two sub-boxes and stored in a list $\mathfrak{L}$ waiting to be treated.

In high dimension, SIVIA is not suitable for a real-time implementation due to the computation time of the bisections. One solution is to use "SIVIAP", a SIVIA with Constraints Propagation $(\mathrm{CP})$ on intervals. CP is very efficient in terms of computation and reduces the number of needed bisections by contracting boxes in order to speed up the processing.

SIVIAP involves the formalization of a Constraint Satisfaction Problem. A CSP is denoted by $\mathcal{H}$ (Eq.1.14) and provides the box $[x]$ that satisfies all the constraints $F$.

$$
C S P:\{\mathcal{H}:(F(x)=\mathbf{0} \mid x \in[x])\}
$$


14Cooperative Localization For Autonomous Vehicles sharing GNSS measurements

Contracting $\mathcal{H}$ means replacing $[\mathbf{x}]$ by a smaller domain $\left[\mathbf{x}^{\prime}\right]$ such that the solution set remains unchanged. The contractors used in this work are ForwardBackward Propagation and Waltz algorithm [33] intersection. More details can be found in [34].

\subsubsection{Cooperative Localization Problem Statement}

Vehicles share their estimated GNSS errors, their DR measurements and their positions. The objective is to get estimates with reliable confidence domains that contain, with high probability, the true positions of the vehicles with little pessimism.

Let us describe the variables, the domains and the constraints of the considered CSP.

\section{Variables}

In each agent $R_{i}$, there is an ego state to be estimated and a tracked position of every known other vehicle $R_{o}$ in the group, where $o \in\left\{1, \ldots, n_{r}-1\right\}$ and $o \neq 0$.

Ego state: let $\nu=\left\{x_{i}, y_{i}, z_{i}, b^{1}, \ldots, b^{n_{s}}, d_{i}, d r_{i}\right\}$ be the variables of the ego state ${ }^{i} \boldsymbol{x}_{\text {ego }}$ of $R_{i}$ of dimension $n=5+n_{s}$. Only these variables are considered in the CSP. Let denote the absolute position by ${ }^{i} \boldsymbol{q}=\left\{x_{i}, y_{i}, z_{i}\right\}$, the biases of all $n_{s}$ satellites in view by ${ }^{i} \boldsymbol{b}=\left\{b^{1}, \ldots, b^{n_{s}}\right\}$ and the inner variables of $R_{i}$ by ${ }^{i} \boldsymbol{\xi}=\left\{d_{i}, d r_{i}\right\}$ which contains respectively the clock offset $d_{i}$ and its drift $d r_{i}$.

Tracked position: let ${ }^{i} \boldsymbol{q}_{o}=\left\{{ }^{i} \boldsymbol{q}_{1}, \ldots,{ }^{i} \boldsymbol{q}_{n_{r}-1}\right\}$ be the positions of the other vehicles estimated by agent $R_{i}$.

The ego input of $R_{i}$ is denoted by ${ }^{i} \boldsymbol{u}_{\text {ego }}=\left\{v_{i}, \psi_{i}\right\}$, where $v_{i}$ and $\psi_{i}$ are respectively the linear speed and the heading angle. ${ }^{i} \boldsymbol{u}_{o}=\left\{{ }^{i} \boldsymbol{u}_{1}, \ldots,{ }^{i} \boldsymbol{u}_{n_{r}-1}\right\}$ represents the input of $R_{o}$ composed of ${ }^{i} \boldsymbol{u}_{o}=\left\{v_{o}, \psi_{o}\right\}$. This information is received from the others.

\section{Domains}

The domains of the variables are sets which enclose the true value of the variables and are represented by boxes, i.e. vectors of intervals of $\mathbb{R}^{n}$ as follows $\left.\left[{ }^{i} \boldsymbol{x}_{\text {ego }}\right]=\left[\begin{array}{lll}{\left[{ }^{i} \boldsymbol{q}\right.} & ]^{T} & {\left[{ }^{i} \boldsymbol{b}\right.}\end{array}\right]^{T}\left[{ }^{i} \boldsymbol{\xi}\right]^{T}\right]^{T}$. Each interval contains the unknown variable $[\nu]=\left[\nu_{\text {true }}-\delta_{\nu}, \nu_{\text {true }}+\delta_{\nu}\right]$, where $\nu_{\text {true }}$ represents the true value of $\nu$ and $\delta_{\nu}$ is the bound of the error.

\section{Constraints}

The constraints that link the variables at each time $k$ are the evolution and observation models:

$$
\begin{gathered}
(i){ }^{i} \boldsymbol{x}_{e g o, k}=\boldsymbol{f}_{k}\left({ }^{i} \boldsymbol{x}_{e g o, k-1},{ }^{i} \boldsymbol{u}_{\text {ego }}\right) \\
(i i){ }^{i} \boldsymbol{y}_{k}=\boldsymbol{g}\left({ }^{i} \boldsymbol{x}_{e g o, k}\right)
\end{gathered}
$$


The constraint (i) corresponds to the dynamic equation of the model, while the constraint (ii) defines the CSP which is used in SIVIAP: ${ }^{i} \boldsymbol{y}_{k}$ are the pseudoranges set to be inverted and ${ }^{i} \boldsymbol{x}_{e g o, k}$ is a prior feasible box coming from a prediction stage.

\section{Solver}

SIVIAP approximates the state vector ${ }^{i} \boldsymbol{x}_{\text {ego }, k}$ such that $\boldsymbol{g}\left({ }^{i} \boldsymbol{x}_{\text {ego }, k}\right) \subseteq\left[{ }^{i} \boldsymbol{y}_{k}\right]$ using a forward-backward contractor. The set to be characterized by SIVIAP is:

$$
\begin{aligned}
C S P & =\left\{{ }^{i} \boldsymbol{x}_{e g o, k} \subseteq\left[{ }^{i} \boldsymbol{x}_{e g o, k}\right] \backslash \boldsymbol{g}\left({ }^{i} \boldsymbol{x}_{e g o, k}\right) \subseteq\left[{ }^{i} \boldsymbol{y}_{k}\right]\right\} \\
& =\boldsymbol{g}^{-1}\left(\left[{ }^{i} \boldsymbol{y}_{k}\right]\right) \cap\left[{ }^{i} \boldsymbol{x}_{\text {ego }, k}\right]
\end{aligned}
$$

\subsubsection{Distributed algorithm}

The same algorithm (1) runs in every vehicle $R_{i}$. Agent $R_{i}$ predicts its ego state ${ }^{i} \boldsymbol{x}_{\text {ego }}$ using the evolution model and its DR inputs $\left(v_{i}, \psi_{i}\right)$ measured at high frequency (line 1). Moreover, it tracks the other vehicles (line 2) using their last received DR inputs.

Lines $(4 \cdots 14)$ of the algorithm consist in updating the predicted state ${ }^{i} \boldsymbol{x}_{e g o}$ with respect to the GNSS measurements which are available every $0.2 s$. In order to reduce the outliers at each time $k$ when the GNSS measurements are available, a validation process on the measurements of every satellite is performed. For every pseudorange measurement, we check if the $S N R$ (Signal to Noise Ratio) of the satellite is high enough (e.g. $35 \mathrm{~dB} / \mathrm{Hz}$ ) and we perform an innovation test based on a punctual estimate with the center of the boxes. Afterward, we apply the SIVIAP algorithm presented in [35] with the following modifications.

The considered solution is the hull box of $\overline{\mathbb{X}}$ which is the union of the inner $\mathbb{X}$ and the indeterminate $\Delta \mathbb{X}$ subpavings as it is shown in Fig 1.3. In order to stop the bisections, we limit the computational time at $0.1 s$ for each epoch. In this case, the tolerable time communication delay is about 100 milliseconds.

In our problem, the vehicles have well synchronized clocks using PPS pulses of the GNSS receivers. The communication delays (line 15) are neglected.

The sent (dataS) and received (dataR) data at time instant $k$ by each vehicle have a unique identifier $i d$ in the group. The amount of the transmitted information on the communication network is low, since vehicles only exchange the lower and upper bounds of the boxes.

In line 16 of the algorithm, each vehicle $i$ updates the tracked position of the $R_{o}$ by the received estimated position $\left[{ }^{i} \boldsymbol{q}_{o}\right]:=\left[{ }^{o} \boldsymbol{q}_{\text {ego }}\right]$. Finally, $R_{i}$ merges its estimated biases with the received ones from the other vehicles $R_{o}$ as follows $\left[{ }^{i} \boldsymbol{b}\right]=\left[{ }^{i} \boldsymbol{b}\right] \cap\left[{ }^{o} \boldsymbol{b}\right]$. 


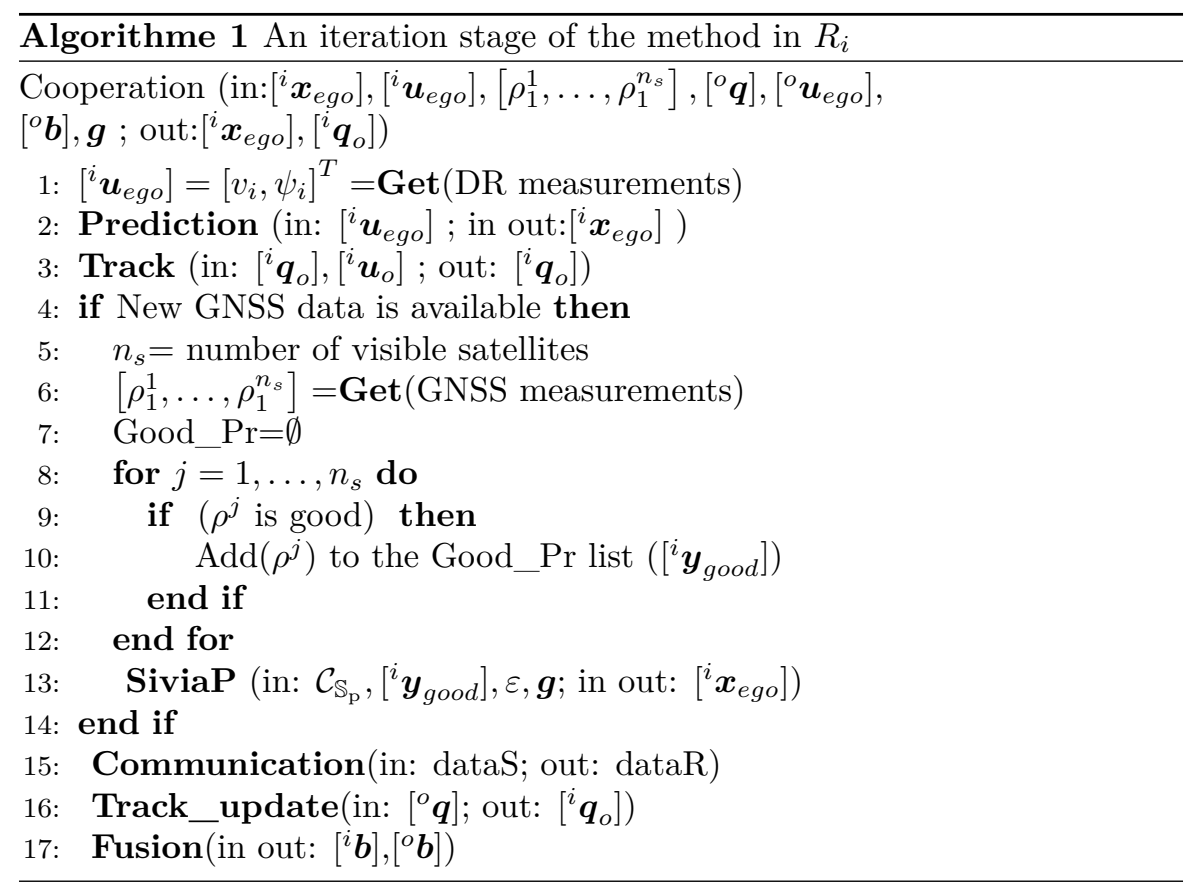

\subsection{Experimental results}

The results of two scenarios, Standalone (S) and Cooperative (C), are reported to quantify the performance gain due to the cooperation using the proposed SIVIAP distributed method. We compare also the SIVIAP estimates with the ones of a rather conventional Bayesian procedure implementing an EKF and involving the same processes: prediction/tracking, update, communication and fusion. Please refer to [28] for more details on the proposed cooperative EKF. The GNSS measurement errors are time correlated and, when doing sequential distributed estimation, the data fusion process that estimates the biases of these measurements incorporate loops. This induces a data incest problem. In the Bayesian framework, a usual method to address this issue is to do the fusion of the biases by using the Covariance Intersection (CI) operator, instead of the Simple Convex Combination (SCC) which is valid only when the errors are uncorrelated. CI is known to provide consistent estimates even when facing an unknown degree of inter-estimate correlation $[36,37]$. In the sequel, we denote by C-SIVIAP and C-EKF-CI the cooperative set-membership and Bayesian methods based on the CI fusion, respectively. 


\begin{tabular}{|c|c|c|}
\hline Variables & & Std. deviation \\
\hline \multirow{2}{*}{$\rho(m)$} & $\mathrm{R} 1$ & $\sqrt{90000.10^{-S N R / 10}}$ \\
\cline { 2 - 3 } & $\mathrm{R} 2$ & 9 \\
\hline \multirow{2}{*}{$v\left({\left.\mathrm{~m} . \mathrm{s}^{-1}\right)}^{-1}\right.$} & $\mathrm{R} 1$ & $1 e-3$ \\
\cline { 2 - 3 } & $\mathrm{R} 2$ & $2 e-3$ \\
\hline \multirow{2}{*}{$\psi\left(\right.$ rad.s $\left.^{-1}\right)$} & $\mathrm{R} 1$ & $2.5 e-3$ \\
\cline { 2 - 3 } & $\mathrm{R} 2$ & $5 e-3$ \\
\hline
\end{tabular}

Table 1.1: Noises parameters used for the estimation methods.

\subsubsection{Experimental setup}

The different approaches have been tested with two experimental vehicles (Fig. 1.2 ) and with the same data-set which was used in a post-processed way. A low-cost U-blox $4 T$ GPS receiver providing raw pseudoranges measurements at $5 \mathrm{~Hz}$ was used in each vehicle. The extraction of broadcast satellite navigation data has been done as follows. Conversion of U-blox navigation data into RINEX files and generation of satellite raw pseudoranges with ionosphere, troposphere, satellite clock offset and time relativity classical corrections. As the localization problem is studied in a local ENU (East-North-Up) frame, the satellite positions, at their emission time, have be transformed into the ENU frame.

In every vehicle, a PolarX Septentrio receiver was used in RTK mode to provide ground truth data with heading $\psi$ information. Indeed, when the receiver is in motion, a GNSS receiver can calculate an accurate track angle which is the measured angle from true North in clock wise direction. When ground vehicles drive with low speed, one can assume that track is equal to heading since slippage can be neglected. A CAN-bus gateway was used to get the linear speed $v$ at $100 \mathrm{~Hz}$ rate. The inputs used by the cooperative system are $\boldsymbol{u}=\left[\begin{array}{ll}v & \psi\end{array}\right]^{T}$.

10 satellites were in view during the test and 5 of them were at least in common which satisfies the necessary condition of the observability study discussed in section 1.2.2. When 4 common satellites between vehicles are not available, one should switch to standalone method. The GPS satellite visibility was sometimes very constrained due to buildings and trees near the test area. Vehicle $R_{2}$ has more satellite in view than vehicle $R_{1}$ during the experiment due to its favorable GNSS environments. The reported test was about 4 minutes long. Fig. 1.4 shows a top view of the trajectories of both vehicles using the $(\mathrm{C})$ set-membership method.

The methods are compared with the same standard deviations parameters presented in Table 1.1. 
18Cooperative Localization For Autonomous Vehicles sharing GNSS measurements

\begin{tabular}{|c|c|c|c|c|}
\hline \multicolumn{2}{|c|}{} & HPE R1 & HPE R2 & Estimated relative distance \\
\hline \hline \multirow{2}{*}{ Std. dev. $(m)$} & $\mathrm{S}$ & 2.28 & 1.58 & 2.87 \\
\cline { 2 - 5 } & $\mathrm{C}$ & 1.71 & 1.53 & 2.58 \\
\hline \multirow{2}{*}{ Max $(m)$} & $\mathrm{S}$ & 5.77 & 5.55 & 8.27 \\
\cline { 2 - 5 } & $\mathrm{C}$ & 6.21 & 4.30 & 8.87 \\
\hline \multirow{2}{*}{ Median $(m)$} & $\mathrm{S}$ & 1.43 & 1.83 & 4.93 \\
\cline { 2 - 5 } & $\mathrm{C}$ & 0.89 & 2.31 & 3.23 \\
\hline
\end{tabular}

\begin{tabular}{|l|c|c|c|}
\hline \multicolumn{2}{|l|}{} & CDS R1 & CDS R2 \\
\hline \hline \multirow{2}{*}{ 95th percentile $\left(m^{2}\right)$} & S & 1652 & 541.4 \\
\cline { 2 - 4 } & C & 475.3 & 256 \\
\hline
\end{tabular}

Table 1.2: $H P E$, relative distance and CDS statistics.

\subsubsection{Set-membership method (SIVIAP) performance analy- sis}

The distributed algorithm has been implemented in $\mathrm{C}++$ using the interval library IBEX [38] and with homemade functions. Fig. 1.4 shows the estimated position boxes of both vehicles. The displayed solution is the hull box $[\overline{\mathbb{X}}]$ of the union of the inner and indeterminate subpavings: $\underline{\mathbb{X}} \cup \Delta \mathbb{X}$.

Fig. 1.5 and 1.6 show the bounds of the position errors of the vehicles respectively for the $x$ and $y$ dimensions using the $(\mathrm{C})$ and $(\mathrm{S})$ methods. At a first glance, one can check the consistency of both observers due to the fact that bounds contain always the zero value. It means that the RTK reference position is always included in the estimated boxes which indicates a good tuning of the observers.

Table 1.2 gives some performance metrics for both methods in term of absolute Horizontal Positioning Error (HPE) and relative distance. The Confidence Domain Size (CDS) of the resulting box is also studied, it is computed with the box volume. The CDS is evaluated via the Cumulative Distribution Function $(\mathrm{CDF})$ of vehicles $2 D$ boxes volumes throughout the trajectory. A net improvement is obtained for vehicle $R_{1}$ in terms of accuracy and confidence. For instance, the median of HPE is reduced from $1.43 \mathrm{~m}$ to $0.89 \mathrm{~m}$ and the CDS is $66.4 \%$ condensed due to the cooperation since the 95th percentile of the CDS is less than $475.3 \mathrm{~m}^{2}$ compared to $1652 \mathrm{~m}^{2}$ when using the S method. Concerning vehicle $R_{2}$, the improvement of HPE is not as significant as in $R_{1}$ since contraction of boxes can move away the center from the reference. The cumulative CDS is $52.7 \%$ reduced since 95th percentile of the CDS is less than $256 \mathrm{~m}^{2}$ compared to $541.4 \mathrm{~m}^{2}$ when using the $\mathrm{S}$ method, which is a substantial improvement.

Regarding the estimation of the relative distance, the method improves the accuracy again trough the fusion of the biases estimates. Especially, if we look at the median and standard deviation errors, they are reduced by $42 \%$ and $20 \%$ respectively which is a significant improvement. 
The bias on every pseudorange has been initialized with the interval $[-30,30]$ (in meters) giving no prior knowledge. For each subplot of Fig. 1.7, the $x$ axis expresses the number of samples, the $y$ axis displays every estimate (the center of the box) of the bias $\left[b^{j}\right]$ with its bounds in meters and $j=1, \ldots, n_{s}$. Note that all subplots are truncated in order to observe the convergence illustrated by the horizontal final asymptotes. This convergence confirms the observability analysis. The obtained final values of the biases are very common for low cost GNSS receivers [39].

Other results illustrating more in details the behavior of the proposed CSIVIAP method can be found in [40].

\subsection{Comparison with a distributed Extended Kalman fil- tering implementing a covariance intersection}

\subsubsection{Backgrounds for Comparison of both methods}

The cooperative estimation of the biases has been implemented with the CI data fusion operator [41]. Eq. (1.16) gives how the covariance matrix and the state estimate are computed.

$$
\left\{\begin{array}{c}
P^{-1}=\omega P_{1}^{-1}+(1-\omega) P_{2}^{-1} \\
\hat{x}=P \cdot\left(\omega P_{1}^{-1} \cdot x_{1}+(1-\omega) P_{2}^{-1} \cdot x_{2}\right)
\end{array}\right.
$$

The weighting factor $\omega \in[0,1]$ has been chosen here to minimize the determinant of the covariance matrix in order to get the smallest uncertainty. The CI fusion provides a conservative and robust result when correlation between two estimates $\hat{x}_{1}$ and $\hat{x}_{2}$ is unknown, as often the case in distributed systems. However, the data fusion algorithm is not optimal [42].

When using a cooperative Bayesian method based on Kalman filtering, vehicles have to share also their estimated error covariance matrix. The used algorithm of the CI fusion of biases estimates is presented in [43]. The weight is the minimum of the covariance matrix determinant of the merged bias errors.

The C-EKF-CI has been implemented with exactly the same data as the CSIVIAP method.

\subsubsection{Comparison criteria}

The choice of good performance metrics is a key issue for assessing a system and evaluating its application. For vehicle localization systems, accuracy is an important metric which usually refers to statistical figures of merit of the 
20 Cooperative Localization For Autonomous Vehicles sharing GNSS measurements

position error. These errors are built with respect to ground truth. For instance, the $95^{\text {th }}$ percentile of the horizontal positioning error distribution can be chosen for accessing the horizontal positioning accuracy. We also propose to evaluate the system reliability by examining the consistencies of the filters. This can be achieved by checking whether the estimated uncertainties correspond to the physical reality of the errors. The confidence bounds of estimated do mains also act as decision variables and so are linked to the pessimism of a localization system. If the confidence bounds are small while keeping the estimates consistent, the localization system is considered to be not too pessimistic. So, a good localization system is a system that provides adequate confidence information and good HPE accuracy. In the following, we use two criteria.

\subsubsection{Horizontal Protection Errors}

The HPE of both methods are defined below:

$$
H P E=\sqrt{e_{x}^{2}+e_{y}^{2}}
$$

where $e_{x}=\widehat{x}-x_{\text {ref }}, e_{y}=\widehat{y}-y_{\text {ref }} \cdot(\widehat{x}, \widehat{y})$ and $\left(x_{\text {ref }}, y_{\text {ref }}\right)$ represent respectively the $2 D$ estimated position and the RTK reference.

For the C-SIVIAP method, the center of the estimated hull box $\left(x_{\text {mid }}, y_{\text {mid }}\right)$ is used as a punctual estimate.

\subsubsection{Confidence Domain Size}

The CDS needs to be assessed to check if the uncertainty is well handled. To gauge this issue, the 2D Cartesian evaluation is transformed in 1D problem by using a statistical distance computation denoted $k \sigma_{H P E}$, where $k$ is the chosen consistency risk according to a $\chi^{2}$ distribution (for a $10^{-2}$ risk, $k=3.035$ this is a common choice that is done here). The $\sigma_{H P E}$ of a Bayesian method is given by Eq. 1.18 [44].

$$
\sigma_{H P E}=\sqrt{\frac{1}{\boldsymbol{u}_{e}^{T} P_{H P E}^{-1} \boldsymbol{u}_{e}}}, \text { with } \boldsymbol{u}_{e}=\left(\begin{array}{c}
e_{x} \\
e_{y}
\end{array}\right) / \sqrt{e_{x}^{2}+e_{y}^{2}}
$$

$\boldsymbol{u}_{e}$ is the unit vector supporting the HPE and $P_{H P E}$ is the estimated matrix of the error covariance when using C-EKF-CI (see Fig. 1.8 for an illustration). For the set-membership C-SIVIAP method, the same consistency $1 \%$ risk $k$ has been considered when setting the bounds on the pseudoranges:

$$
\left[\rho_{i}^{j}\right]=\left[\rho_{i}^{j}-k \sigma_{\rho}, \rho_{i}^{j}+k \sigma_{\rho}\right]
$$

where $j$ represents the satellite index and $\sigma_{\rho}$ represents the standard deviation of the pseudorange measurement presented in table 1.1.

In way similar to the Bayesian method, the CDS of C-SIVIAP is defined by a $k \sigma_{H P E}$ segment in the direction of the HPE vector, as shown by figure 1.9. 
Comparison with a distributed Extended Kalman filtering implementing a covariance intersection 21

\subsubsection{Comparison Results}

\subsubsection{Accuracy analysis}

Figure 1.10 depicts the cumulative distribution of HPEs of the both aforementioned methods. $95 \%$ of the HPE errors for vehicle $R_{1}$ are less than $10.46 \mathrm{~m}$ and $5.32 m$, respectively for C-EKF-CI and C-SIVIAP. For vehicle $R_{2}$ these figures are less than $7.64 \mathrm{~m}$ and $4.41 \mathrm{~m}$ respectively. The accuracy gain of CSIVIAP compared to C-EKF-CI is $49.13 \%$ for $R_{1}$ and $42.27 \%$ for $R_{2}$. The set-membership approach is clearly more accurate than the Bayesian one.

\subsubsection{Consistency analysis}

Let us consider now the filters consistency which is checked if the CDS bounds actually the HPE i.e. $H P E<k \sigma_{H P E}$. Consistency tests using HPEs are fundamental to provide suitable horizontal protection levels (HPL) for cooperative vehicles [45].

Figure 1.11 presents $2 D$ histograms in order to evaluate the consistency of the two methods for vehicles $R_{1}$ and $R_{2}$. The horizontal and vertical axis represent respectively the HPE and the CDS (i.e. $k \sigma_{H P E}$ ) computed for each navigation solution. Each pixel tabulates the total number of occurrences of a specific (HPE, CDS) pair. Note that the color scale is logarithmic. These histograms can be considered as simplified Stanford diagrams since we are only interested in regions where we have CDS $>\mathrm{HPE}$ and HPE $>$ CDS. Points where the CDS is under the HPE error indicate a failure of integrity. In this way, the gray area corresponds to overconfident outcomes of the filters.

It appears from these results that the two methods are $100 \%$ consistent since there is no point in the gray area. Therefore, C-EKF-CI and C-SIVIAP methods are both reliable in the sense that the ground truth is always included in the estimated confidence domain.

As confidence is in practice compared to a threshold to indicate "use" or "don't use" to the client application, it is important, in terms of availability of the positioning information, to provide as small as possible confidence domains. Let us look especially at the $k \sigma_{H P E}$ of both methods for each vehicle in figure 1.11 .

It can be observed that the confidence domains produced by the C-SIVIAP method are significantly tighter than the C-EKF-CI ones, in particular for vehicle $R_{2}$.

This indicates that the bounded-error method significantly reduces uncertainties compared to a Bayesian method based on covariance intersection fusion.

To resume the comparison analysis of the methods, the consistencies of setmembership and Bayesian filters are achieved. Both methods provide reliable confidence domains that contain the true positions of vehicles. Reliability is quite important for navigation missions in approach in order to avoid collision 
problem. However, the cooperative set-membership method performs better than the Bayesian one in terms of accuracy and uncertainty as it gives a significant improvement of positioning accuracy and a good decrease of the confidence domains.

If we look at performance comparison between the two vehicles when using the set-membership method, it can be observed that the best performance is obtained for vehicle $R_{2}$ which has more accurate positions (i.e, lower HPE) and less uncertainties (i.e, smaller CDS). This is due to the fact that $R_{1}$ has less satellites in view and its DR sensors are of less good quality compared to vehicle $R_{2}$.

\subsection{Conclusion}

This work has presented two cooperative localization methods for intelligent vehicles sharing GNSS common errors. It has been found that at least 4 satellites and an auto-regressive model of the biases are needed to keep the states observable which means that the problem is solvable even if there is no fixed base station well located. The proposed cooperative bounded error approach based on set inversion method with constraint propagation provides a significant enhancement in terms of accuracy and confidence domains compared to usual standalone methods.

The reuse of identical information (which are here the estimated biases) in the fusion process is also naturally managed by a set-membership approach. Such kind of method therefore deals correctly with the data incest issue. Moreover, it handles rigorously the non-linearities of the equations. We have also reported a performance comparison of the bounded-error method with a cooperative sequential Bayesian approach based on Kalman filtering and on covariance intersection fusion of the biases estimates. The experimental results indicate that both methods give reliable confidence domains of vehicles positions. However, the set-membership approach has the advantage to provide more accurate positions with smaller confidence domains. The key information deduced from this comparison is that set-membership methods are very suitable for applications requiring high integrity/accuracy in cooperative navigation contexts.

In future work, the robustness of the tuning of the proposed method should be more deeply considered by conducting different tests. This is important to address complex situations as urban canyons where simultaneous multipath issues are frequent. Moreover, the proposed algorithm has been designed to have a period higher that the communication delay between vehicles. In a V2X network, the transmission of real-time data with a guaranteed latency is not possible (due to collision issues for instance). In this case, a solution is to 
keep a time-stamped data buffer in each vehicle and to process the data in a sliding time window after the received data have been placed at the correct place in the buffer. The position is updated in the past and propagated up to the current time. 
- 


\section{Bibliography}

[1] S. I. Roumeliotis and G. A. Bekey. Collective localization: a distributed kalman filter approach to localization of groups of mobile robots. In IEEE International Conference on Robotics and Automation (ICRA), volume 3, pages 2958-2965 vol.3, 2000.

[2] A. Martinelli, F. Pont, and R. Siegwart. Multi-robot localization using relative observations. In IEEE International Conference on Robotics and Automation (ICRA), pages 2797-2802, April 2005.

[3] S. I. Roumeliotis and I. M. Rekleitis. Analysis of multirobot localization uncertainty propagation. In IEEE/RSJ International Conference on Intelligent Robots and Systems (IROS), volume 2, pages 1763-1770 vol.2, Oct 2003 .

[4] E. D. Nerurkar, S. I. Roumeliotis, and A. Martinelli. Distributed maximum a posteriori estimation for multi-robot cooperative localization. In IEEE International Conference on Robotics and Automation (ICRA), pages 1402-1409, May 2009.

[5] A. Howard, M. J. Matark, and G. S. Sukhatme. Localization for mobile robot teams using maximum likelihood estimation. In IEEE/RSJ International Conference on Intelligent Robots and Systems (IROS), volume 1, pages 434-439 vol.1, 2002.

[6] L. Montesano, J. Gaspar, J. Santos-Victor, and L. Montano. Cooperative localization by fusing vision-based bearing measurements and motion. In IEEE/RSJ International Conference on Intelligent Robots and Systems (IROS), pages 2333-2338, Aug 2005.

[7] Y. Lin, P. Vernaza, J. Ham, and D. D. Lee. Cooperative relative robot localization with audible acoustic sensing. In IEEE/RSJ International Conference on Intelligent Robots and Systems (IROS), pages 3764-3769, Aug 2005.

[8] S. Worrall and E. Nebot. Using non-parametric filters and sparse observations to localise a fleet of mining vehicles. In IEEE International Conference on Robotics and Automation (ICRA), pages 509-516, April 2007. 
[9] H. Mu, T. Bailey, P. Thompson, and H. Durrant-Whyte. Decentralised solutions to the cooperative multi-platform navigation problem. IEEE Transactions on Aerospace and Electronic Systems, 47(2):1433-1449, April 2011.

[10] S. I. Roumeliotis and G. A. Bekey. Distributed multirobot localization. IEEE Transactions on Robotics and Automation, 18(5):781-795, Oct 2002 .

[11] Elwan Héry, Philippe Xu, and Philippe Bonnifait. Along-track Localization for Cooperative Autonomous Vehicles. In IEEE Intelligent Vehicles Symposium (IV 2017), pages 511-516, Redondo Beach, CA, United States, Jun 2017.

[12] R. Madhavan, K. Fregene, and L. E. Parker. Distributed heterogeneous outdoor multi-robot localization. In IEEE International Conference on Robotics and Automation, volume 1, pages 374-381 vol.1, 2002.

[13] H. Kruppa D. Fox, W. Burgard and S.Thrun. A probabilistic approach to collaborative multi-robot localization. Autonomous Robots, 8(3):325-344, 2000 .

[14] A. Howard, M. J. Mataric, and G. S. Sukhatme. Putting the 'i' in 'team': an ego-centric approach to cooperative localization. In IEEE International Conference on Robotics and Automation (ICRA), volume 1, pages 868-874 vol.1, Sept 2003.

[15] N. Karam, F. Chausse, R. Aufrere, and R. Chapuis. Localization of a group of communicating vehicles by state exchange. In IEEE Int Conf on Intelligent Robots and Systems, pages 519-524, Oct 2006.

[16] M. Kieffer. Estimation ensembliste par analyse par intervalle : Application à la localisation de véhicules. PhD thesis, Université de Paris Sud, 1999.

[17] Michel Kieffer and Eric Walter. Interval analysis for guaranteed nonlinear parameter and state estimation. Mathematical and Computer Modelling of Dynamical Systems, 11(2):171-181, 2005.

[18] L. Jaulin. A nonlinear set membership approach for the localization and map building of underwater robots. IEEE Trans. on Robotics, 25(1):8898, Feb 2009.

[19] L. Jaulin. Range-only SLAM with occupancy maps ; a set-membership approach. IEEE T-RO, 27(5):1004-1010, 2011.

[20] Jaulin L. and Walter E. Set inversion via interval analysis for nonlinear bounded-error estimation. Automatica, 29(4):1053-1064, 1993. 
[21] V. Drevelle and Ph. Bonnifait. Localization Confidence Domains via Set Inversion on Short-Term Trajectory. IEEE Trans on Robotics, 29(5):1244-1256, 2013.

[22] D. Meizel, O. Leveque, L. Jaulin, and E. Walter. Initial localization by set inversion. IEEE Trans on Robotics and Automation, 18(6):966-971, 2002 .

[23] L. Jaulin. Interval constraint propagation with application to boundederror estimation. Automatica, 36(10):1547-1552, 2000.

[24] A. Bethencourt. Interval Analysis for swarm localization. Application to underwater robotics. PhD thesis, University of Bretagne, Sept 2014.

[25] Kyoung-Hwan Jo and Jihong Lee. Cooperative localization of multiple robots with constraint propagation technique. In IEEE Int Conf on Intelligent Robots and Systems, pages 3477-3482, Sept 2008.

[26] P. Bonnifait K. Lassoued and I. Fantoni. Cooperative Localization with Reliable Confidence Domains Between Vehicles Sharing GNSS Pseudoranges Errors with No Base Station. IEEE Intelligent Transportation Systems Magazine, 9(1):2234, 2017.

[27] E. D. Kaplan and C. Hegarty. Understanding GPS: Principles and Applications. Second Edition, Artech House, 2005.

[28] K. Lassoued, I. Fantoni, and P. Bonnifait. Mutual localization and positioning of vehicles sharing GNSS pseudoranges: Sequential bayesian approach and experiments. In IEEE Int Conf on Intelligent Transportation Systems, Spain, Sept 2015.

[29] X. Xiao J. Rife. Estimation of spatially correlated errors in vehicular collaborative navigation with shared GNSS and road-boundary measurements. In Int Technical Meeting of The Satellite Division of the Institute of Navigation, pages 1667-1677, Sept 2010.

[30] R. Hermann and A.J. Krener. Nonlinear controllability and observability. IEEE Trans on Automatic Control, 22(5):728-740, Oct 1977.

[31] A. Martinelli and R. Siegwart. Observability analysis for mobile robot localization. In IEEE Int Conf on Intelligent Robots and Systems, pages 1471-1476, Aug 2005.

[32] X.S. Zhou and S.I. Roumeliotis. Robot-to-robot relative pose estimation from range measurements. IEEE Trans on Robotics, 24(6):1379-1393, Dec 2008.

[33] D. Waltz. Generating semantic descriptions from drawings of scenes with shadows. The psychology of computer vision, 1975. 
[34] K. Lassoued, O. Stanoi, Ph. Bonnifait, and I. Fantoni. Mobile robots cooperation with biased exteroceptive measurements. In Int Conf on Control Automation Robotics Vision, pages 1835-1840, Singapore, Dec 2014.

[35] L. Jaulin, M. Kieffer, O. Didrit, and E. Walter. Applied interval analysis with examples in parameter and state estimation, robust control and robotics. Springer, 2001.

[36] H. Li and F. Nashashibi. Cooperative multi-vehicle localization using split covariance intersection filter. IEEE Intelligent Transportation Systems Magazine, 5(2):33-44, Summer 2013.

[37] CY. Chong and S. Mori. Convex combination and covariance intersection algorithms in distributed fusion. In 4th International Conference on Information Fusion, Canada, 2001.

[38] G. Chabert. IBEX(http://www.ibex-lib.org), 2007.

[39] O. Le Marchand, Ph. Bonnifait, J. Ibanez-Guzman, D. Betaille, and F. Peyret. Characterization of gps multipath for passenger vehicles across urban environments. ATTI dell'Istituto Italiano di Navigazione, (189):77-88, 072009.

[40] K. Lassoued, P. Bonnifait, and I. Fantoni. Cooperative localization of vehicles sharing gnss pseudoranges corrections with no base station using set inversion. In IEEE Intelligent Vehicles Symposium (IV), Gothenburg, Sweden, June 19-22 2016.

[41] N. R .Ahmed, S. J . Julier, J. R . Schoenberg, and M. E . Campbell. Multisensor Data Fusion From Algorithms and Architectural Design to Applications, chapter Decentralized Bayesian Fusion in Networks with Non-Gaussian Uncertainties, pages 383-408. CRC Press, 2015.

[42] U. W. Utete. Network Management in Decentralized Sensing Systems. $\mathrm{PhD}$ thesis, Department of Engineering Science, University of Oxford, 1995.

[43] K Lassoued. Localisation de robots mobiles en coopération mutuelle par observation d'état distribuée. $\mathrm{PhD}$ thesis, Université de Technologie de Compiègne, Sorbonne universités, 2016.

[44] V. Drevelle and $\mathrm{Ph}$. Bonnifait. Localization confidence domains via set inversion on short-term trajectory. IEEE Transactions on Robotics, 29(5):1244-1256, 2013.

[45] M. Worner, F. Schuster, F. Dolitzscher, C. G. Keller, M. Haueis, and K. Dietmayer. Integrity for autonomous driving: A survey. In IEEE/ION Position, Location and Navigation Symposium (PLANS), pages 666-671, April 2016. 


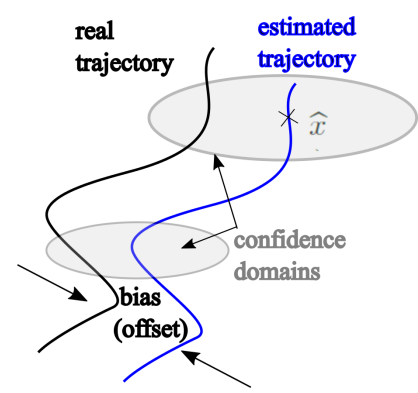

Figure 1.1: Illustration of the effects of biased measurements. Confidence domains are zones in which the vehicle is highly located.

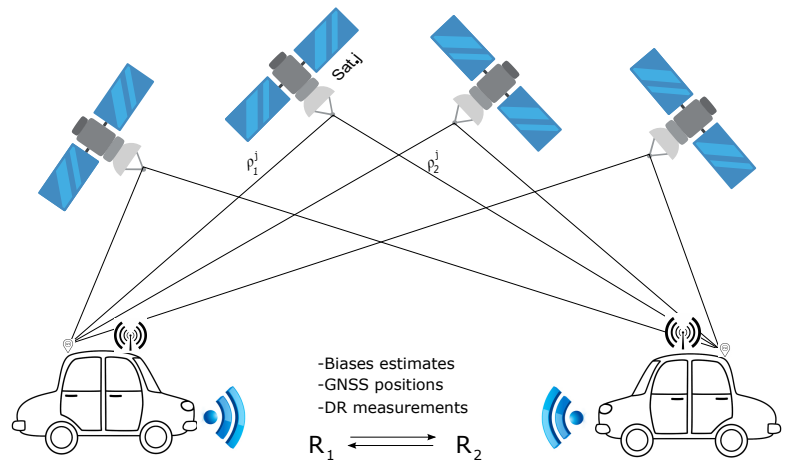

Figure 1.2: Experimental vehicles and shared data 


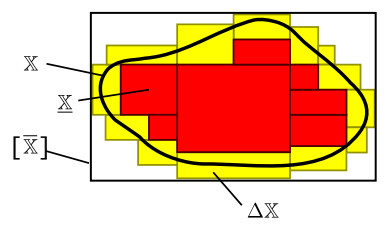

Figure 1.3: Bracketing of the solution set $\mathbb{X}$ between two subpavings $\underline{\mathbb{X}}$ and $\overline{\mathbb{X}}=$ $\mathbb{X} \cup \Delta \mathbb{X} \cdot[\overline{\mathbb{X}}]$ is the hull box of $\overline{\mathbb{X}}$.

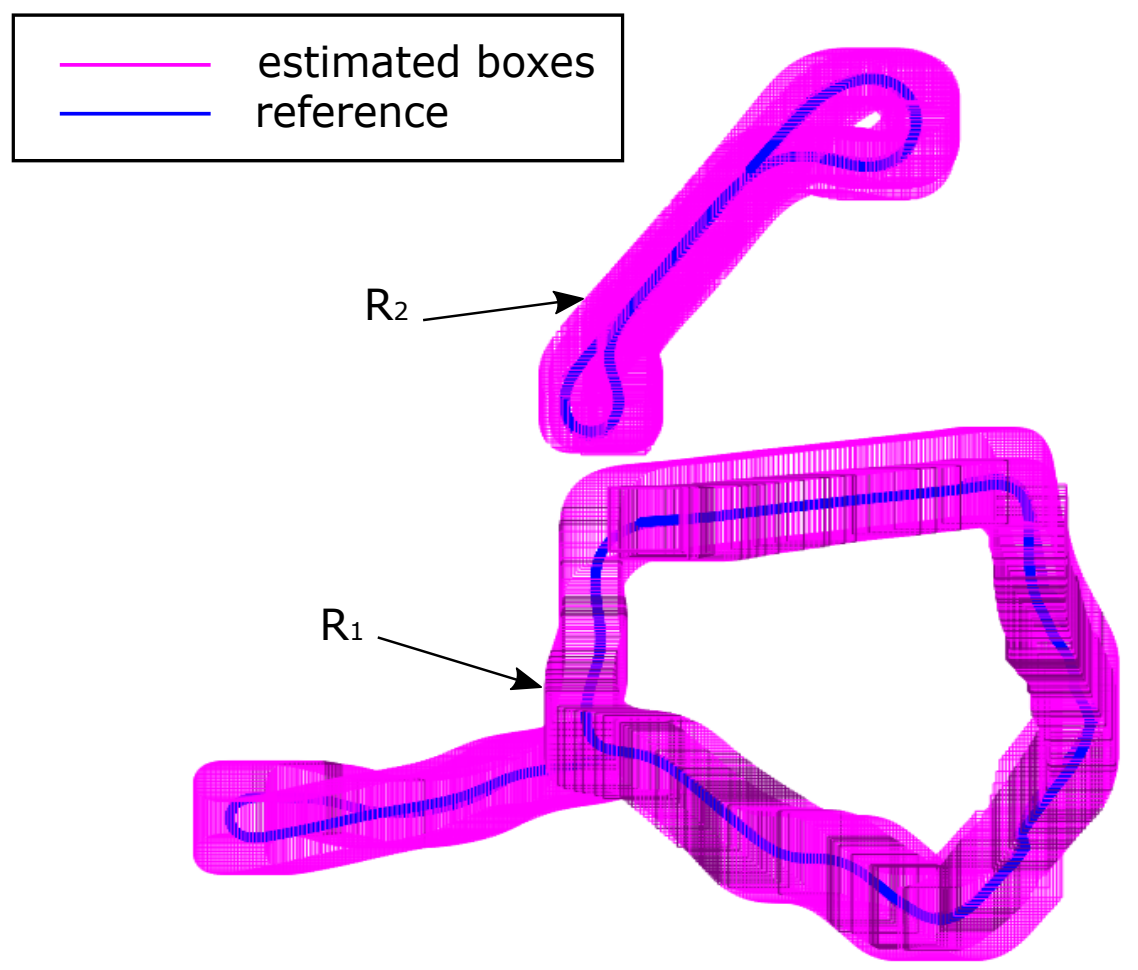

Figure 1.4 : Trajectories of both vehicles when using C-SIVIAP. Reference and position boxes are displayed. The mean speed of vehicles was $30 \mathrm{Kmph}$. Every vehicle did several loops of its trajectory. 

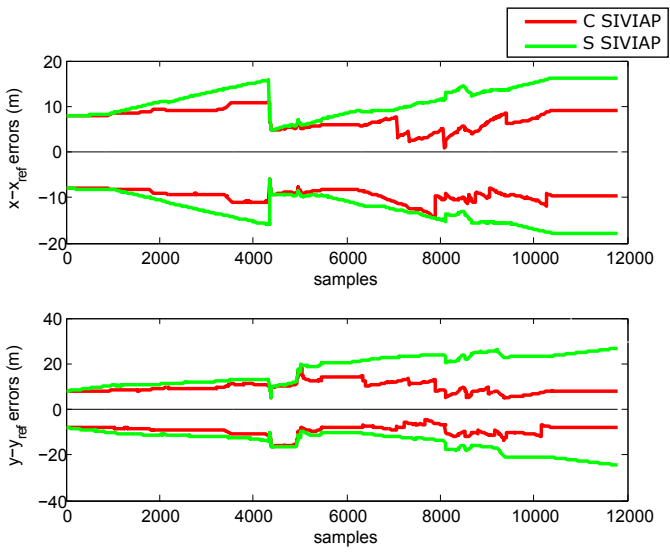

Figure 1.5 : Bounds of position errors of $R_{1}$ centered on the reference.
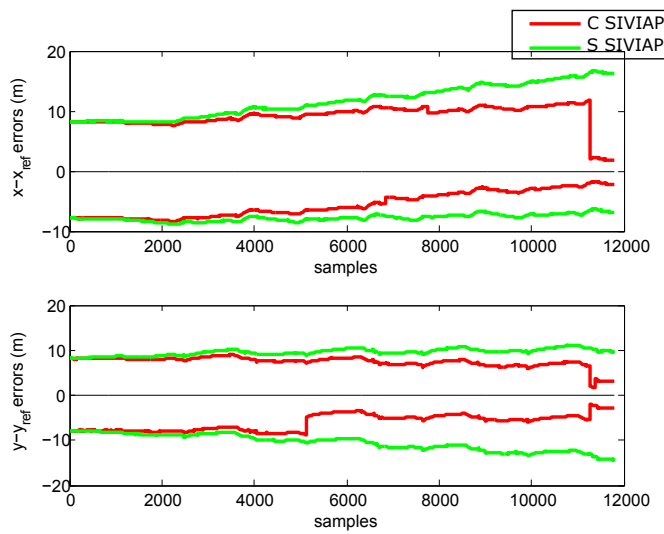

Figure 1.6 : Bounds of position errors of $R_{2}$ centered on the reference. 

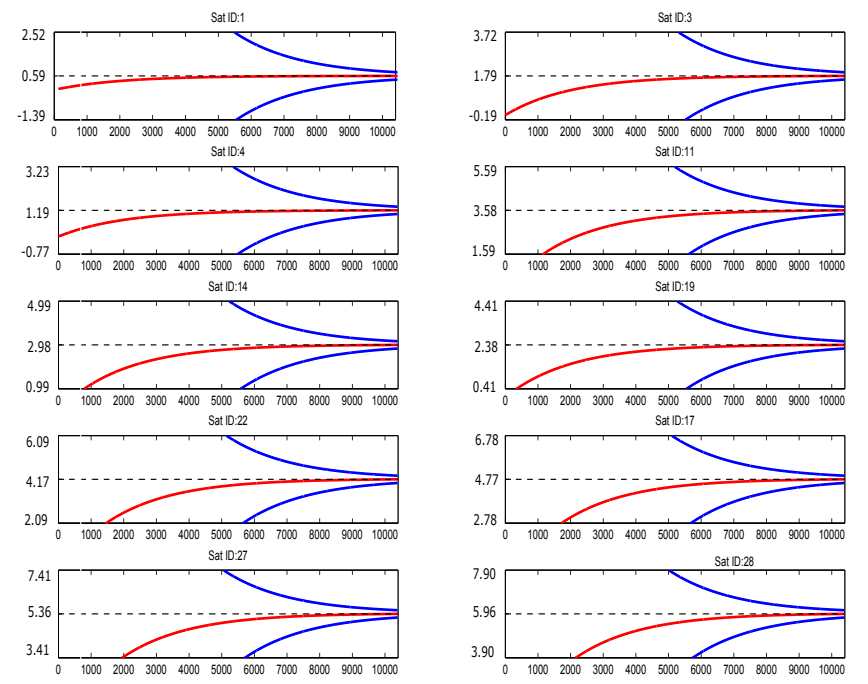

Figure 1.7 : Estimates and bounds of pseudoranges biases (units are samples and meters).

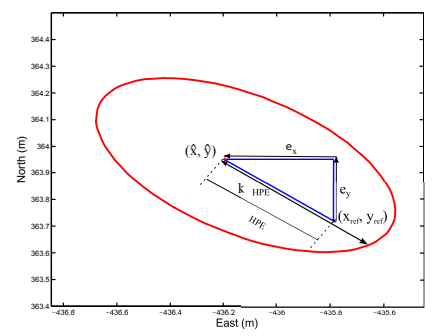

Figure 1.8 : HPE and CDS illustration for C-EKF-CI.

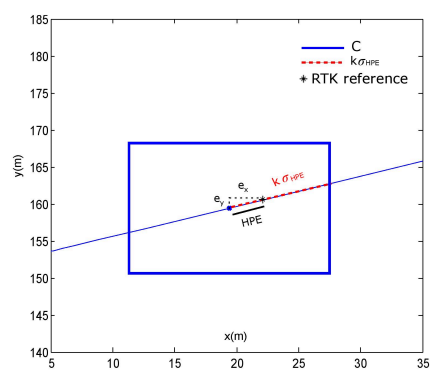

Figure 1.9 : HPE and $k \sigma_{H P E}$ illustration for $C$-SIVIAP. 


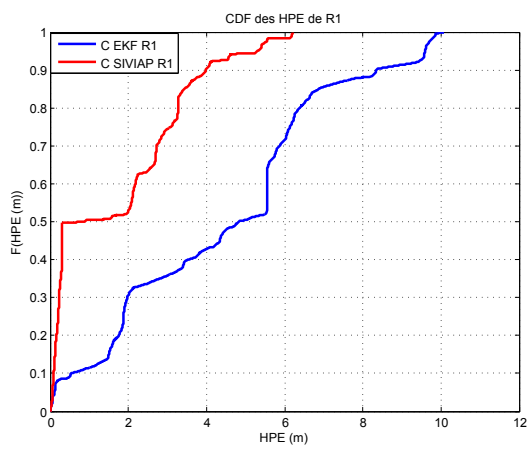

(a) Cumulative HPE of $R_{1}$.

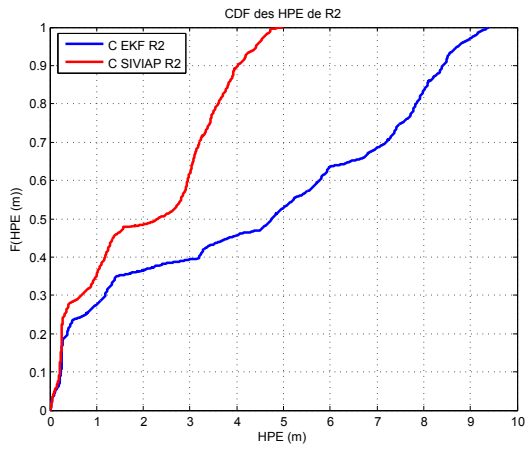

(b) Cumulative HPE of $R_{2}$.

Figure 1.10 : Plots of the cumulative distribution function (CDF) of the HPE produced by the two methods in the two vehicles (C EKF : cooperative EKF based on CI fusion, C SIVIA : cooperative set-membership method). 


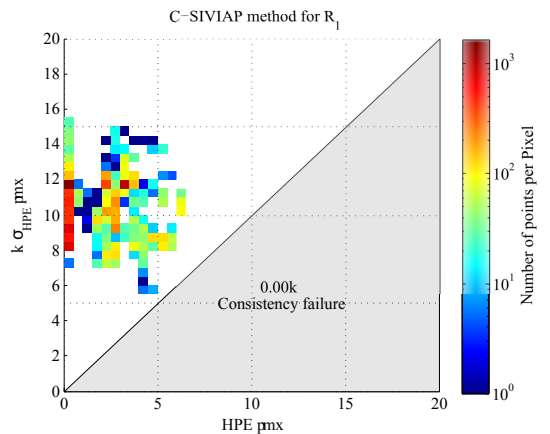

(a) C-SIVIAP for $R_{1}$.

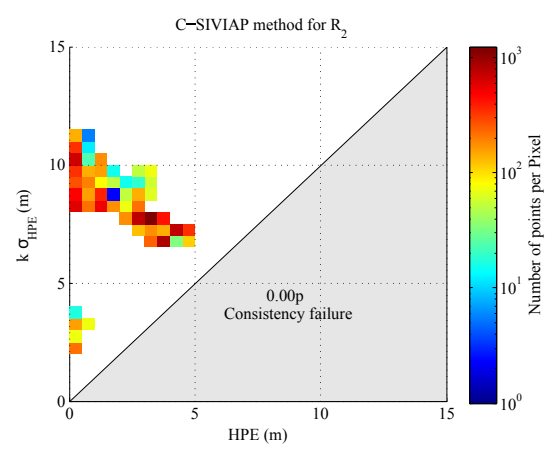

(c) C-SIVIAP for $R_{2}$.

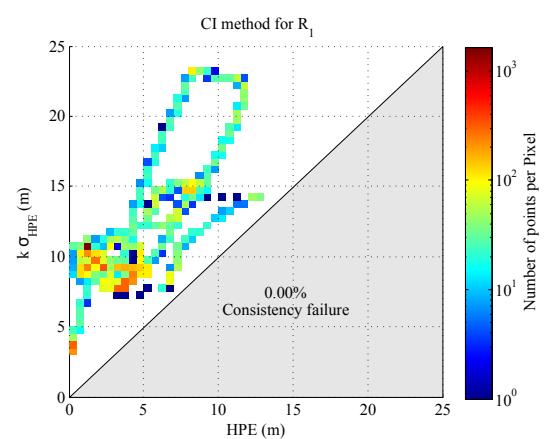

(b) $C$-EKF-CI for $R_{1}$.

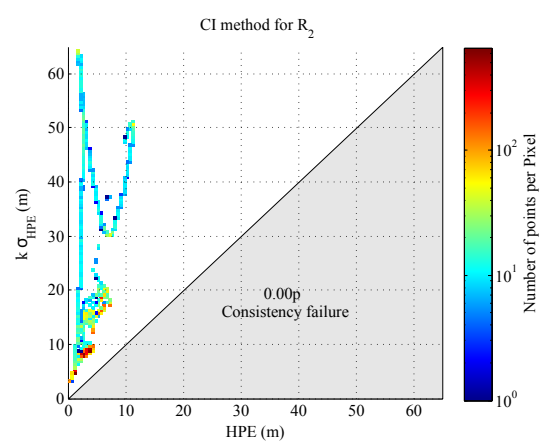

(d) C-EKF-CI for $R_{2}$

Figure 1.11 : simplified Stanford Diagram : Filters consistency when using cooperative set-membership and Bayesian methods for $R_{1}$ and $R_{2}$. (a) C-SIVIAP for $R_{1}$, (b) $C$-EKF based on CI fusion for $R_{1}$, (c) C-SIVIAP for $R_{2}$, (d) C-EKF based on CI fusion for $R_{2}$. 


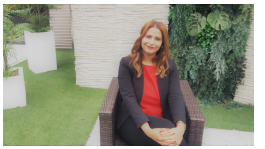

Khaoula Lassoued is a research and development engineer for the Autonomous Transport Systems Department of Transdev Group in France. She graduated as an automatic electric engineer from the ENIG School in Tunisia, then succeeded in a research Masters thesis in the DRIVE Laboratory of the University of Burgundy. Since 2016, she has obtained her PhD in Autonomous Vehicle Localization and Computer Science at the Heudiasyc laboratory, Université de Technologie de Compiègne (UTC), which is a member of the Sorbonne. Her research topics include Robotics and autonomous vehicles software and sensors specifically.

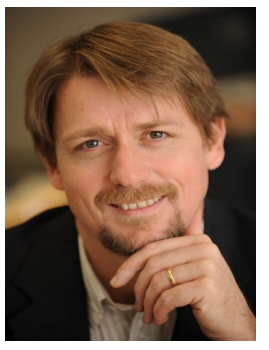

Philippe Bonnifait is a professor in the Computer Science department of the Université de Technologie de Compiègne (UTC), France. He obtained his Ph.D. degree in automatic control and computer science from the école Centrale de Nantes in 1997. Since 1998, he has been with Heudiasyc UMR 7253, a common research laboratory between UTC and CNRS. His research interests are in Intelligent Vehicles and Robotics. He is currently working on high integrity positioning methods for autonomous systems and self-driving vehicles. 